\title{
Der optimale Schutz vor Zahnerosion
}

Zahnerosion wird unter anderem durch den häufigen Konsum von säurehaltigen Nahrungsmitteln und Getränken, wie Limonade und Obst verursacht. Sie sind auch bei guter Mundhygiene möglich und nicht reversibel. Mittlerweile ist jeder 3. Erwachsene von Zahnerosion betroffen Tendenz weiter steigend. Aber: Noch immer findet dieses Problem viel zu wenig Beachtung. Dabei gibt es Möglichkeiten, dem Verlust der Zahnhartsubstanz entgegenzuwirken.

Die elmex ${ }^{\circledR}$ EROSIONSSCHUTZ Zahnpasta von CP GABA mit ihrer ChitoActive-Technologie enthält Chitosan (ein Biopolymer natürlichen Ursprungs), Zinnchlorid und Aminfluorid. Diese Kombination bildet eine schützende, zinnreiche Schicht auf der Zahnoberfläche, wobei Zinn-Ionen in den aufgeweichten Zahnschmelz eingela- gert werden. Der Zahnschmelz wird so resistenter gegen erosive Säureangriffe und gegen den Abbau von aufgeweichtem Zahnschmelz. Die ChitoActive-Technologie ist zum Patent angemeldet.

Für eine effektive Reinigung und erhöhten Schutz sollte elmex EROSIONSSCHUTZZahnpasta wie gewohnt im Rahmen der täglichen Mundhygiene angewendet werden. Die Ergebnisse einer randomisierten, doppelt blinden klinischen In-situ-Studie im Cross-over-Design mit 27 Probanden zeigen einen signifikant höheren Schutz vor Erosion gegenüber einer natriumfluoridhaltigen Zahnpasta (Schlueter $\mathrm{N}$ et al. Caries Res 2013; 47: 574-581).
Für optimalen Schutz vor ZahnschmelzAbbau empfiehlt sich die Anwendung der Zahnpasta in Kombination mit elmex EROSIONSSCHUTZ-Zahnspülung und elmex ZAHNSCHMELZ-SANFT Zahnbürste. Diese Zahnbürste reinigt durch optimal abgerundete Reinigungsborsten und extra sanfte Filamente mit mikrofeinen Enden gründlich aber schonend bis in die Zahnzwischenräume.

Nach einer Pressemitteilung der

CP GABA GmbH, Hamburg 